\title{
Hospital medicine beyond the United States
}

This article was published in the following Dove Press journal: International Journal of General Medicine

\section{Flora Kisuule \\ Eric Howell}

Johns Hopkins School of Medicine, Division of Hospital Medicine, Johns Bayview Medical Center, Baltimore, MD, USA
Correspondence: Flora Kisuule Johns Hopkins School of Medicine, Division of Hospital Medicine, Johns Bayview Medical Center, 5200 Eastern Avenue, MFL West, 6th Floor, Baltimore, MD 2I224, USA

Email fkisuul I@jhmi.edu

\begin{abstract}
Hospital medicine is the fastest growing specialty in the United States. An interesting aspect of the rapid expansion of hospital medicine is the expansion of the field beyond the United States. Although the health care systems, regulations, and cultural norms in these nations differ, there are striking similarities in the profession's development. We performed a literature review to better understand the factors contributing to the growth of hospital medicine internationally. In this article, we describe some of the drivers for expansion of hospital medicine outside the United States and the challenges faced by these groups. We also discuss the role the United States could play in the continued growth of hospital medicine internationally.
\end{abstract}

Keywords: international hospital medicine

\section{Introduction}

Hospital medicine is the fastest growing specialty in the United States. In 2016, this specialty celebrated the 20th anniversary of the term "hospitalist". ${ }^{1,2}$ Current estimates put the number of hospitalists at over $50,000 .^{2}$ Generalist training including internal medicine, family medicine, medicine-pediatrics, and pediatrics formed the basis of most hospitalist programs during the initial 15 years of the specialty; however, today, several subspecialties are involved in hospitalist programs. ${ }^{3}$ An interesting aspect of the rapid expansion of hospital medicine is the growth of the field beyond the United States. The Society of Hospital Medicine (SHM) reports more than 15,000 North American members and an additional 126 international hospitalist members (Ethan Gray, Vice President of Membership, SHM, personal communication, August 30, 2017). ${ }^{4}$ Although the health care systems, regulations, and cultural norms in these nations differ, some of the reasons for the development of hospital medicine internationally are the same as in the United States. ${ }^{1,5}$ In this article, we focus on adult hospital medicine. We describe some of the drivers for expansion of this field beyond the United States and the challenges faced by these groups. We also speculate on the future of hospital medicine internationally and discuss the role that the United States could play in the continued growth of this specialty beyond its borders.

\section{Drivers for the exportation of hospitalist model to Canada}

Canada is the first case of the expansion of hospital medicine beyond the United States, and as of 2008, Canada had more than 100 hospital medicine programs. ${ }^{7}$ 
Currently, the estimated number of hospitalists in Canada has increased to $~ 3,000$ (Colleen Savage, Administrator, Canadian SHM, personal communication, December 6, 2017). Yousef and Maslowski describe several drivers for the development of the hospitalist model, which are related to physicians, patients, and systems. Work-life balance and the desire for non-hospital work among primary care providers (PCPs) were leading physician factors. Two major patient-related factors were the increasing age and complexity of patients and the increasing number of "unattached" patients. Unattached patients are those who either do not have a PCP or their PCP does not have admitting hospital privileges. System drivers included PCP shortages, reduction in resident duty hours, higher need for health system efficiency, and cost reduction. Further, increasing health system complexity led to PCPs withdrawing from hospital care. ${ }^{7}$

\section{Drivers for the exportation of the hospitalist model beyond North America}

The success of the hospitalist model in North America, especially related to improving quality, reducing costs, and optimizing hospital operations, has resulted in the exportation of the hospitalist models across the globe. The SHM reports members and attendees at the hospital medicine annual meeting in 2017 representing hospitalist programs from 33 different countries excluding the United States and Canada (Figure 1). Specific international drivers of hospitalist growth are discussed in Table 1.

\section{Suboptimal coordination of inpatient care}

In Singapore, general practitioners (GPs) do not attend on their patients when they are admitted to the hospital. The care of patients is handed over to specialists, sometimes many specialists, with the potential for conflicting treatment plans and limited or no clear coordination. For example, a patient with heart failure and acute kidney injury might have

Table I Drivers for and barriers against the establishment of hospital medicine programs beyond North America

\begin{tabular}{l} 
Drivers \\
Suboptimal coordination of care \\
Health care costs \\
Globalization \\
Patient safety and quality improvement \\
Patient experience \\
Health care access \\
Capacity and throughput \\
Culture \\
Barriers \\
Training \\
Productivity and efficiency \\
Care transitions \\
Compensation \\
Stigma and mistrust \\
\hline
\end{tabular}

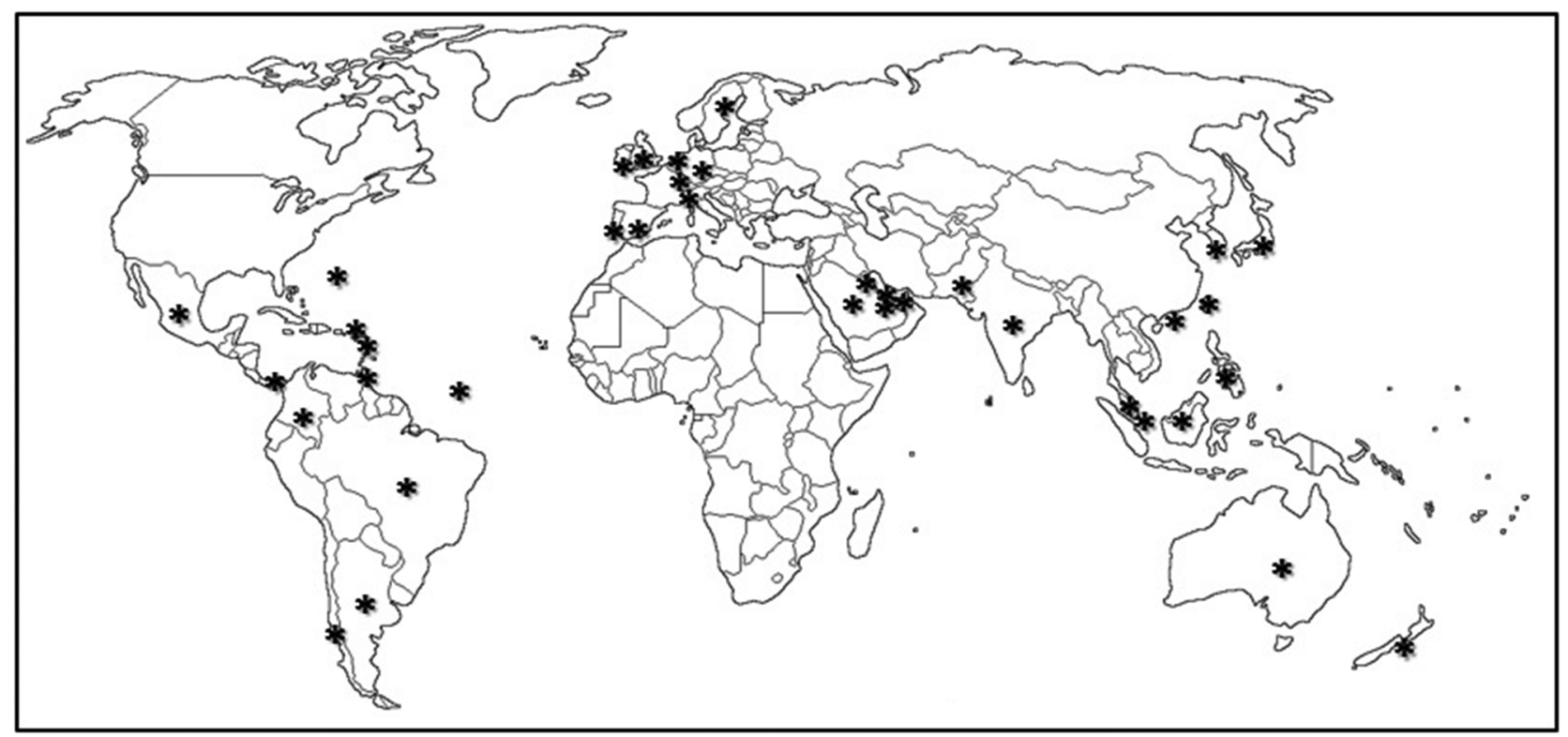

Figure I Current active Society of Hospital Medicine (SHM) international membership and representation at SHM annual meetings in 2016 and 2017 by country. Notes: *Argentina, Aruba, Bahrain, Belgium, Bermuda, Brazil, Columbia, Germany, Grenada, Hong Kong, India, Ireland, Japan, Korea, Kuwait, Malaysia, Mexico, Netherlands, New Zealand, Panama, Pakistan, Portugal, Qatar, Saint Kitts, Saudi Arabia, Singapore, Spain, Sweden, Switzerland, Taiwan, United Arab Emirates, United Kingdom, and Virgin Islands. (C) 2009 www.outline-world-map.com. 
recommendations for diuresis and intravenous (IV) fluids, resulting in conflicting treatment plans. This lack of care coordination can potentially be mitigated by the hospitalist model. ${ }^{7}$ Similarly, in Switzerland, there was the need to integrate specialist care provided in hospitals so as to reduce costs and improve quality. Some Swiss hospitals started employing "hospital specialists" whose main responsibility is to manage patients autonomously and coordinate care. ${ }^{8}$

\section{Health care costs}

Taiwan developed a National Health Insurance (NHI) that was implemented in 1995, which extended coverage to almost the entire population. This availability of coverage resulted in increased hospitalizations. Shu et al performed a study where they compared a hospitalist-run ward to an internist-run ward at a medical referral center and found that the hospitalist system had higher efficiency with reduced costs and improved length of stay (LOS). Their conclusion was that the hospitalist model may serve as an alternative model to address increasing admissions, staff shortages, and costs. ${ }^{9}$

\section{Globalization}

Globalization is defined as, "[...]]the process of international integration arising from the interchange of world views, products, ideas and other aspects of culture [... ". ${ }^{10}$ Schroth and Khawaja"1 wrote that "highly trained physicians and struggling public health systems that are unable to meet the needs and expectations of patients are some of the forces behind the demand for globalization in healthcare". The need to identify best global practices has resulted in partnerships between institutions in the United States and hospitals around the world (Kisuule et al, 2014, unpublished). As a part of the joint venture between Johns Hopkins International and Saudi Aramco, a thriving hospitalist group has been developed at Johns Hopkins Aramco Health in Dhahran, Kingdom of Saudi Arabia (KSA) (Kisuule et al, 2014, unpublished). Weill Cornell Medical College has partnerships with Hamad Medical Corporation in Doha, Qatar, which is home to a thriving hospitalist practice. ${ }^{12}$ Cleveland Clinic Abu Dhabi has established a hospital medicine division. They have extended their US-based model of care to the United Arab Emirates (UAE) so as to provide access to care, including hospitalization, without the need for patients to travel abroad for treatment. ${ }^{13}$

\section{Patient safety and quality improvement}

In Chile, historically, hospitalized patients are predominately managed by independent specialists doing rota- tions at the hospital. In this model, there is no physician ownership of quality improvement within those hospitals despite a strong desire nationally to build a culture of quality and safety. Hospital Clinico Universidad de Chile has implemented a hospitalist program. That hospitalist group has now developed protocols for deep vein thrombosis prophylaxis, glycemic management, and pneumonia management and has been funded to carry out safety research for government. ${ }^{14}$

In Panama, general and specialist physicians are under financial stress and work at multiple hospitals and clinics to make ends meet. This has led to off-site coverage of inpatients. There was an urgent need to ensure on-site 24/7 physician coverage, leading to the development of a hospitalist program at Hospital Punta Pacifica in Panama City (Kisuule and Lowe, 2015, unpublished).

\section{Patient experience}

Interest in improving the patient experience is not just a phenomenon in the United States. ${ }^{15,16}$ In Taiwan, private hospitals with occupancy rates of $40 \%-60 \%,{ }^{9}$ a low rate compared to their US counterparts, compete for patients. There is a need to ensure that patients are happy with the care they receive to capture market share. In addition to reducing costs and improving quality, Taiwan has seen the added benefit of improved patient satisfaction when they explored the hospitalist model. ${ }^{9}$ In Chile, at Hospital Clinico de la Pontificia Universidad Catolica de Chile, the hospitalist service saw their patient satisfaction surveys improve since the advent of the hospitalist service. Patients indicated that a driver of that satisfaction was that the hospitalists were on-site and available more often than the traditional physician practice. $^{14}$

\section{Hospital capacity and throughput}

In Taiwan, NHI coverage increased the number of patients in the emergency department (ED) leading to overcrowding and patients boarding for more than 72 hours. Shu et al showed that hospitalists reduced inpatient LOS, which improved hospital capacity and helped alleviate ED overcrowding. ${ }^{9}$ Hospitalist groups in institutions such as Johns Hopkins Aramco Health are utilizing hospitalist in triage attending roles for ED admissions (Kisuule et al, 2014, unpublished). Studies in the United States have long demonstrated that hospitalist-driven bed management enhances the bed utility rate. ${ }^{16-18}$ Therefore, it is not surprising that international hospitalist programs have tackled ED throughput as well. 


\section{Culture}

In some countries, there is stigma attached to being a generalist and primary care practitioner. For example, in Brazil, low pay and stigma leads many generalist physicians into subspecialty practice. However, there is a growing trend among younger physicians in Brazil to find hospital medicine as an appealing career. ${ }^{14}$ Interviews conducted at the site visit at Hospital Punta Pacifica in Panama showed that the newly graduated medical doctors wanted to go into some subspecialty because general medicine did not pay well. Becoming a hospitalist provided an alternative to the training required for subspecialization and would command respect and better pay (Kisuule and Lowe, 2017, unpublished).

\section{Challenges faced by hospitalist programs internationally \\ Training}

There are different types of training models for hospitalist groups around the world. In Australia, traditional medical officers who have completed 6 years of medical school and 1 year of internship work under the supervision of boardcertified physicians. These medical officers have been reclassified as hospitalists. In addition to previous training, they receive faculty, career, and educational development, and hence, they can function more independently. ${ }^{14}$ The skill set of these providers has been elevated, and hence, they can be involved in quality improvement and the operations of a hospital. ${ }^{14}$ In Saudi Arabia, current practice is to recruit hospitalists who are North American trained and are board certified to practice in the United States or Canada. It is not easy to recruit such physicians, and the result is often long periods of time when the hospitalist service is understaffed (Kisuule et al, 2014, unpublished). In addition, physicians who have completed their training in Saudi or other Arab countries are employed as "ward physicians" and function in a capacity similar to physician assistants in the United States. They do not have admitting privileges and work collaboratively with a board-certified attending. In Panama, hospitalists are licensed GPs who have completed 6 years of medical school and 2 years of practical training (internship). After the 2 years of training, the doctors obtain a certificate and can practice independently, but only as an outpatient GP in Panama. Once licensed as GPs, doctors can engage in specialty training. The only hospitalist model currently existing in Panama utilizes licensed GPs. However, as noted, these GPs cannot see inpatients independently (Kisuule and Lowe, 2017, unpublished). Hospital Punta Pacifica is working to establish the first true hospitalist program in Panama by hiring internists to staff the hospitalist service (Kisuule and Lowe, 2017, unpublished). The University of Panama Medical School is considering developing a master's program in hospital medicine so as to provide additional training to GPs, so that they can eventually practice independently in the hospital setting (Kisuule and Lowe, 2015, unpublished).

\section{Productivity and efficiency}

In the United Kingdom, and other commonwealth countries, inpatients are taken care of by a consultant-led team where the daily "hands on care" is done by junior doctors. In such systems, efficiency may be difficult to realize as there are delays in care because the junior doctors have to consult the supervising physician prior to making any patient management decisions. For example, at Tawam Hospital in the UAE, the hospitalist service consists of consultant-led teams who practice in the UK model described above (Kisuule and Panek, 2015, unpublished). These teams include interns, residents, and junior doctors who are referred to as "specialists" (not to be confused with traditional specialists who have competed additional training such as a fellowship). Junior doctors have completed residency and are Arab board certified. Although these junior doctors could practice independently, they are still included on these teams. In these consultant-led systems, there may be a need to develop parallel services where consultants or junior doctors engage in direct patient care. These hospitalists, particularly if considered junior physicians, would need to have autonomy to make patient decisions independently.

There are cultural competing interests that can be counter intuitive to hospitalist goals. For example, hospitalists aim to reduce LOS and readmissions; however, in certain cultures, it is challenging to do so. In the UAE and KSA, hospitalizations can last weeks due to family preference without actual clinical necessity. A provider needs the patient's agreement before discharge. Shu et al describe that hospitalizations in Japan and Taiwan last for 1-3 weeks. ${ }^{19}$

\section{Care transitions}

Many countries around the globe do not have long-term care facilities. Alternatively, long-term care facilities may exist, but in very limited numbers. Patients may be ready to leave the acute hospital setting; however, there may be no long-term care or rehabilitation facilities for the patients to go to after discharge. As a result, these patients stay in the hospital. Situations such as these make it hard for hospitalists to start, or grow, a program because external forces such as these make it hard to show their value. The traditional metrics 
that hospitalists are able to impact in the United States, for example, lower LOS, reduced readmissions, or reduced costs, are harder to move in some of these international settings because of inadequate care transition options (Kisuule et al, 2014, unpublished; Kisuule and Panek, 2015, unpublished).

\section{Compensation}

In Panama, physicians are not paid very well so they rotate at multiple hospitals to make ends meet (Kisuule and Lowe, 2015, unpublished; Kisuule and Lowe, 2017, unpublished). This is also true in Chile and Brazil where physicians at public hospitals are paid poorly, so they round for a few hours in the public hospitals and then go to work in the private hospitals and clinics. ${ }^{14,20}$ This makes it hard to develop a hospitalist model in the public sector.

\section{Stigma and mistrust}

In Panama, the general doctors, who also practice as hospitalists, do not command the respect of some of the specialists because they do not have specialty training and are perceived by patients, specialists, and hospital administrators not to have the skill set to manage patients independently. In South Korea, inpatient care is provided by specialists who follow their patients from clinic to the inpatient setting. It is not uncommon for an inpatient to be taken care of by multiple specialists unless the reason for hospitalization is related to one organ system (Joong-Sik E, Jang S, Yoo C, personal communication, May 5, 2016). Two main barriers to establish hospitalist groups in South Korea are concerns by specialists that the hospitalists will "steal their patients" and concerns that the hospitalists, who are non-specialized internists by training, may not be well qualified to adequately care for the patients (Joong-Sik E, Jang S, Yoo C, personal communication, May 5, 2016). This stigma and mistrust are compounded by the fact that hospital medicine is still a new field in many parts of the world.

\section{Role the United States could play in the growth of hospital medicine internationally}

The United States now has more than 20 years of experience in the field of hospital medicine. Hospitalists in the United States have been tapped to establish international hospitalist groups or to support and improve existing groups (Kisuule et al, 2014, unpublished; Kisuule and Lowe, 2015, unpublished; Kisuule and Lowe, 2017, unpublished; Kisuule and Panek, 2015, unpublished). In many parts of the world, hospitalists and hospital medicine are unknown, and some of these infant groups are struggling to define themselves and prove their worth (Kisuule and Lowe, 2015, unpublished; Kisuule and Lowe, 2017, unpublished). Organizations such as the SHM, the American College of Physicians (ACPs), and the Society of General Internal Medicine (SGIM) can help support these groups by helping them to validate themselves. This could be done through establishing international SHM chapters and helping them organize regional conferences. For example, in the Middle East, Qatar held its third internal medicine conference in October 2016. During this conference, there was a workshop supported by SHM. In South America, there is the Pan American Society of Hospitalists, which holds conferences and has hosted renowned US hospitalists as key note speakers. The ACP has chapters in many of the countries that now have hospitalist groups and could include hospitalists as speakers at their regional meetings. Continued efforts to support these international groups, as they organize, will be key in seeing continued growth of hospital medicine around the globe.

The benefits of hospital medicine such as improved quality and reduced costs would be of great value to the developing world. However, these are the areas where establishing hospitalist program is most challenged. Doctors who are straight out of school are not completely qualified to take care of patients independently by regulation. Yet, these "young" doctors are the ones who could most easily transition into the hospitalist role. The US-based hospitalist institutions, particularly those with medical education backgrounds or fellowship programs, could help train these doctors by developing online modules or by developing relationships where training opportunities such as grand rounds could be broadcast internationally. SHM, ACP, and other subspecialty organizations have several educational modules that could be made available, at an affordable cost, to these budding hospitalists.

In addition to the need for more training, in some countries, low salaries are a great hindrance to doctors working at just one hospital. Many physicians make ends meet by practicing in multiple hospitals and clinics. Hospitalists in the United States have shown their ability to provide high value care. If these benefits could be reproduced beyond the United States, hospitals with hospitalists could potentially see an increase in their market share, which could potentially lead to higher hospitalist salaries. This could potentially result in hospitals supplementing physician salaries. Currently, in the United States, $50 \%$ of a hospitalist's salary is supported by the hospital in which they work.

Improved patient safety and reduced costs have been the driver for regulatory bodies in countries such as the UAE to 
seek to establish hospitalist programs. There is a need for these international hospitalist groups to prove their worth to their hospitals and governments. SHM, ACP, and hospitalist groups have developed care pathways, protocols, and implementation toolkits that improve efficiency and quality of care. Efforts could be made to facilitate the dissemination of these best practices internationally.

Finally, government and regulatory buy-in are crucial for the continued growth of hospital medicine internationally. Hospitalists in the United States have a track record of securing such buy-in. For example, hospitalist can now recertify with a focus in hospital medicine, have testified in congress, and have influenced policies that affect the country. Leaders in our field could lend their expertise to some of these new groups in working with their governments to make this a recognized specialty in their countries.

\section{Conclusion}

Hospital medicine has grown rapidly since its inception in the mid-1990s. The success and rapid growth of this specialty have been driven by hospitalists showing their value through improved patient safety and quality, improved patient experience, and reduced costs. All these factors have led to an interest in, and the exportation of, the hospitalist model. The United States has seen a rapid increase in the number of hospitalists in the past 20 years. Many of the drivers for the development of hospital medicine internationally are the same as those in the United States. Yet, the growth of hospital medicine internationally faces unique challenges around culture, training, and compensation. Hopefully, US hospitalists will partner with their international counterparts to ameliorate some of these barriers, so that hospital medicine can see the same success as it has in the United States.

\section{Disclosure}

The authors report no conflicts of interest in this work.

\section{References}

1. Wachter RM, Goldman L. The emerging role of "hospitalists" in the American health care system. N Engl J Med. 1996;335(7):514-517.
2. Wachter RM, Goldman L. Zero to $50,000-T$ The $20^{\text {th }}$ anniversary of the hospitalist. N Engl J Med. 2016;375:1009-1011.

3. Kisuule F, Howell E. Hospitalists and their impact on quality, patient safety, and satisfaction. Obstet Gynecol Clin N Am. 2015;42: 433-446.

4. Society of Hospital Medicine [homepage on the Internet]. Available from: https://www.hospitalmedicine.org/Web/Membership/Web/Membership/Membership_Landing_Page.aspx. Accessed Tuesday April 11, 2017.

5. Messler J, Whitcomb W. A history of the hospitalist movement. Obstet Gynecol Clin N Am. 2015;42:419-432.

6. Yousefi V, Maslowski R. Health system drivers of hospital medicine in Canada. Can Fam Physician. 2013;59:762-767.

7. Hock Lee K, Yang Y, Soong Yang K, Chi Ong B, Seong Ng H. Bringing generalists into the hospital: outcomes of a family medicine hospitalist model in Singapore. J Hosp Med. 2011;6:115-121.

8. Burkhardt U, Erbsen A, Rudiger-Sturcher M. The hospitalist as coordinator: an observational case study. J Health Organ Manag. 2010;24(1):22-44.

9. Shu CC, Lin JW, Lin YF, Hsu NC, Ko WJ. Evaluating the performance of a hospitalist system in Taiwan: a pioneer study for nationwide health insurance in Asia. J Hosp Med. 2011;6:378-382.

10. Globalization [webpage on the Internet]. Wikipedia; 2016. Available from: https://en.wikipedia.org/wiki/Globalization. Accessed July 18, 2016.

11. Schroth L, Khawaja R. Globalization of healthcare. Front Health Serv Manage. 2007;24(2):19-30.

12. Weill Cornell Medicine-Qatar [homepage on the Internet]. Available from: https://qatar-weill.cornell.edu. Accessed August 23, 2017.

13. Cleveland Clinic Abu Dhabi [homepage on the Internet]. Available from: https://www.clevelandclinicabudhabi.ae/en/about-us/pages/default. aspx. Accessed October 14, 2016.

14. Berthold J [webpage on the Internet]. Hospital medicine around the world. From the July 2009 ACP Hospitalist. Available from: http:// www.acphospitalist.org/archives/2009/07/world.htm. Accessed July 18, 2016.

15. Wray CM, Flores A, Padula WV, Prochaska MT, Meltzer DO, Arora VM. Measuring patient experiences on hospitalist and teaching services: patient responses to a 30-Day Postdischarge Questionnaire. J Hosp Med. 2016;11(2):99-104.

16. Howell E, Bessman E, Marshall R, Wright S. Hospitalist bed management effecting througuphut from the emergency department to the intensive care unit. J Crit Care. 2010;25:184-189.

17. Howell E, Bessman E, Kravet S, Kolodner K, Marshall R, Wright S. Active bed management by hospitalists and emergency department throughput. Ann Intern Med. 2008;149:804-811.

18. Soong $\mathrm{C}$, Wright $\mathrm{S}$, Kisuule $\mathrm{F}$, et al. The role of hospitalists in managing patient flow: lessons from four hospitals. Curr Emerg Hosp Med Rep. 2016;4(4):159-163.

19. Shu CC, Hsu NC, Lin YF, Wang JY, Lin JW, Ko WJ. Integrated postdischarge transitional care in a hospitalist system to improve discharge outcome: an experimental study. BMC Med. 2011;9:96.

20. Beresford L. Hospitalists around the world. Hospitalist. 2015;19(1): $27-30$. 
The International Journal of General Medicine is an international, peer-reviewed open-access journal that focuses on general and internal medicine, pathogenesis, epidemiology, diagnosis, monitoring and treatment protocols. The journal is characterized by the rapid reporting of reviews, original research and clinical studies across all disease areas.
The manuscript management system is completely online and includes a very quick and fair peer-review system, which is all easy to use. Visit http://www.dovepress.com/testimonials.php to read real quotes from published authors. 